\title{
Política de cohesión y fondos estructurales de la Unión Europea: retos y perspectivas para el período post-2020
}

\author{
European Union structural funds and cohesion policy: \\ challenges and prospects for the post-2020 period
}

\author{
Beatriz Pérez de las Heras \\ Directora de Cuadernos Europeos de Deusto \\ beatriz.perez@deusto.es
}

doi: http://dx.doi.org/10.18543/ced-58-2018pp17-22

Resumen: Con una dotación de 351.800 millones de euros en el actual marco financiero plurianual 2014-2020, la política de cohesión de la Unión Europea (UE) encara actualmente un proceso de revisión cara a su redefinición en el próximo marco financiero posterior a 2020. La negociación de este nuevo horizonte de financiación afronta diversos desafíos, como la salida del Reino Unido, uno de los mayores contribuyentes al presupuesto de la UE, la crisis migratoria y la seguridad exterior de la UE. El futuro de la política de cohesión dependerá de los resultados de esas negociaciones, en las que ya se vislumbran algunas propuestas concretas, como la simplificación de la burocracia administrativa o el mejor uso y gestión de las herramientas financieras. Las contribuciones de este número monográfico de Cuadernos Europeos de Deusto abordan algunos de estos desafíos y perspectivas que planean actualmente sobre la política de cohesión de la UE, aportando distintos elementos de análisis y reflexión sobre su futura evolución.

Palabras clave: política de cohesión, fondos estructurales, marco financiero plurianual post-2020.

Abstract: With an endowment of EUR 351,800 million in the current 20142020 multiannual financial framework, the European Union (EU) cohesion policy is currently undergoing a revision process with a view to its redefinition in the next, post-2020 financial framework. Negotiation on this new funding horizon faces several challenges, such as the United Kingdom's exit from the EU, one of the biggest contributors to the EU budget, the migratory crisis and the EU's external security. The future of the cohesion policy will depend on the results of those negotiations. Some concrete proposals are already envisioned, such as the simplification of the administrative bureaucracy or the better use and management of financial tools. The contributions of this special issue of Cuadernos Europeos de Deusto address some of these challenges and perspectives that are cur- 
rently being discussed about the EU's cohesion policy while providing different elements for further analysis and reflection on its future evolution.

Keywords: cohesion policy, structural funds, post-2020 multiannual financial framework.

La política de cohesión de la Unión Europea (UE) constituye un pilar clave del proceso de integración en Europa. Como principal política de inversión de la UE, su objetivo es impulsar el crecimiento económico, la competitividad, el desarrollo sostenible y la calidad de vida de los ciudadanos en todas las regiones y ciudades de la UE. Implementada principalmente a través de tres fondos estructurales, a saber, el Fondo de Cohesión, el Fondo Social Europeo, el Fondo de Desarrollo Regional, la política de cohesión ha servido de catalizador del apoyo financiero público y privado para proyectos que han reforzado la dimensión urbana, la inclusión social, las inversiones directas en pequeñas y medianas empresas o el medio ambiente, entre otros. Más allá de su relevancia económica y social, todos estos logros contribuyen, sin duda, a generar atracción y confianza en el proyecto político europeo en su conjunto.

Con una dotación de 351.800 millones de euros en el actual marco financiero plurianual 2014-2020, la política de cohesión encara actualmente un proceso de revisión cara a su redefinición en el próximo marco financiero posterior a 2020. La negociación de este nuevo horizonte de financiación afronta diversos desafíos, como la salida del Reino Unido, uno de los mayores contribuyentes al presupuesto de la UE, la crisis migratoria y la seguridad exterior de la UE. El futuro de la política de cohesión dependerá de los resultados de esas negociaciones, en las que ya se vislumbran algunas propuestas concretas, como la simplificación de la burocracia administrativa o el mejor uso y gestión de las herramientas financieras.

Las contribuciones de este número monográfico de Cuadernos Europeos de Deusto abordan algunos de estos desafíos y perspectivas que planean actualmente sobre la política de cohesión de la UE, aportando distintos elementos de análisis y reflexión sobre su futura evolución.

En primer lugar, los Profesores de la Universidad de Salamanca, Rafael Bonete Perales, Profesor Titular de Economía Aplicada, y Rafael Muñoz de Bustillo Llorente, Catedrático de Economía Aplicada, ofrecen un estudio detenido sobre la reforma de la política de cohesión cara al nuevo marco financiero plurianual en curso de negociación. Su análisis incluye, así mismo, una mirada sobre los efectos potenciales que la reconsideración de esta política puede suponer para la región de Euskadi. 
Después de un repaso a la evolución global de la cohesión territorial en la UE y a los elementos que se debaten sobre el futuro de la política de cohesión, el artículo concluye que si esta política es dotada de menos fondos y de un carácter territorialmente más concentrado, como se discute actualmente, Euskadi podría resultar desfavorecida por los ajustes. Como alternativas, los autores proponen aprovechar al máximo los recursos del Fondo Europeo para Inversiones Estratégicas, del Plan Juncker de inversiones, las posibilidades financieras que ofrece el Banco Europeo de Inversiones (BEI) y el programa COSME para mejorar la competitividad de las pequeñas y medianas empresas, así como las que ofrecerá el nuevo programa de I+D que reemplace a HORIZONTE a partir de 2021.

Javier Bilbao Ubillos, Catedrático de Economía Aplicada en la Universidad del País Vasco, aborda en su artículo la dimensión social de la política de cohesión, un aspecto de gran relevancia, no sólo en el contexto de revisión en curso de esta política, sino también en el momento actual de reflexión sobre el futuro de la UE. Partiendo de la evolución de la razón de ser de la política de cohesión, inicialmente denominada «política regional», como acción dirigida a corregir las disparidades de renta entre regiones, el Profesor Bilbao analiza el impacto que sucesivos programas y estrategias de cohesión han tenido en términos de dimensión social. Concluye el autor que a pesar de los innegables logros conseguidos con respecto a la mejora de las condiciones de vida y empleo de los ciudadanos, el énfasis y enfoque de la política de cohesión se centra en los territorios y no en las personas. Su objetivo primordial ha sido siempre reducir las disparidades regionales. Con todo, la convergencia regional se ha ralentizado en estos últimos años de crisis económica y deficiente gestión macroeconómica. Todo ello hace que las políticas de promoción social sigan desarrollándose esencialmente a nivel nacional, a la espera de que la UE asuma mayores competencias y compromisos en materia social.

\section{Roberto San Salvador del Valle, Geana de Miranda Leschko y} Milica Matovic, investigadores de la Universidad de Deusto, se adentran en la evolución del lugar y papel que las ciudades han tenido en el proceso de integración europea en general y en el marco de la política de cohesión, en particular. Argumentan los autores que desde el primer programa URBAN (1994) hasta el Pacto de Amsterdam y la Agenda Urbana (2016), el protagonismo de las ciudades en Europa no ha hecho sino acrecentarse. Esta mayor importancia de la dimensión urbana forma parte de una tendencia global por la que las ciudades se han 
transformado en los núcleos donde se concentra más de la mitad de la población mundial y epicentro, al mismo tiempo, de los grandes desafíos globales (cambio climático, seguridad energética, transportes y movilidad, etc). Concluyen los autores que la Agenda Urbana de la UE deberá servir de guía en el proceso de reforma de la política de cohesión y en el contexto del nuevo marco financiero plurianual (2021-2027). Así mismo, en consonancia con los compromisos internacionales asumidos por la UE y sus Estados miembros, en esa redefinición también habrá que tenerse en cuenta la Agenda de Desarrollo Sostenible 2030 y la Nueva Agenda Urbana de Naciones Unidas. Para ello, proponen un modelo transversal y transectorial, en el que las ciudades emerjan como «ecosistemas de innovación transformadora de la Unión Europea y del Mundo».

Christopher Huggins, Doctor en Relaciones Internacionales e Investigador en la Universidad de Aberdeen (Reino Unido), aporta un análisis sobre los efectos que tendrá para las autoridades locales inglesas la salida del Reino Unido de la UE y la consecuente pérdida de acceso a los fondos estructurales. En concreto, el autor analiza en primer lugar el papel, relativamente escaso, jugado por las autoridades locales durante el referéndum británico de junio de 2016. Destaca a continuación, como en el contexto actual de negociaciones con la UE, las autoridades locales están implicándose más activamente reclamando al Gobierno británico fuentes alternativas de financiación a las europeas. El autor concluye que, por el momento, las demandas locales permanecen desatendidas, lo cual no es sino reflejo de la incertidumbre que rodea las negociaciones sobre el Brexit, a lo que se añade como dificultad adicional una tendencia de centralización creciente en el sistema político inglés.

En la sección de Otros Estudios, Carlos Espaliú Berdud, Profesor Agregado de Derecho Internacional Público y de la Unión Europea de la Universidad Internacional de Cataluña, nos ofrece un análisis de las operaciones navales de la UE en el marco de la Política Común y de Seguridad Común, valorando su contribución a una defensa europea más integrada.

Finalmente, concluyen este número 58/2018 las habituales crónicas de jurisprudencia europea, de nuestro colaborador David Ordoñez Solís, Magistrado y miembro de la Red de Expertos en Derecho de la Unión Europea del Consejo General del Poder Judicial, y de actualidad europea, de nuestra colaboradora Beatriz Iñarritu Ibarretxe, Profesora Doctora de la Deusto Business School. 
A todos los colaboradores de este número, expresamos nuestro agradecimiento sincero por sus valiosas contribuciones, así como a la Diputación Foral de Bizkaia que, a través de su Dirección General de Acción Exterior, patrocina y apoya la edición de Cuadernos Europeos de Deusto desde hace más de 20 años.

\section{Sobre la autora}

Beatriz Pérez de las Heras es Catedrática de Derecho de la Unión Europea y Titular de la Cátedra Jean Monnet en Integración Europea en la Universidad de Deusto (UD). Doctora en Derecho por la UD, realizó estudios de postgrado en el Centre Européen Universitaire de Nancy, donde obtuvo los diplomas de DESS y DEA en Droit Communautaire. Fue Directora del Instituto de Estudios Europeos de la UD, de 1996 a 2009, así como Coordinadora Académica del Centro de Excelencia Jean Monnet en Derecho de la Unión Europea y Relaciones Internacionales, de 2013 a 2016. Desempeñó, así mismo, el puesto de Vicedecana de Investigación y Relaciones Internacionales en la Facultad de Derecho, de 2013 a 2015. Ha realizado diversas estancias como investigadora visitante en las Universidades americanas de Oregon (2009), Georgetown (2012), Fordham (2013), Boston College (2014) y Florida International University (2016). Actualmente dirige la revista Cuadernos Europeos de Deusto y es Investigadora Principal del equipo de investigación Integración Europea y Derecho Patrimonial en un contexto global. Es autora de numerosas publicaciones sobre cuestiones jurídicas europeas, entre artículos en revistas científicas y algunos libros, como Hacia un Derecho Común en la Unión Europea (Dykinson, 2003) y El Mercado Interior Europeo (Universidad de Deusto, 2008). Sus líneas de investigación actuales se centran principalmente en la política de cambio climático y de energía de la Unión Europea (UE), democracia y ciudadanía en la UE, y el papel de la UE como actor internacional.

\section{About the author}

Beatriz Pérez de las Heras is Professor of European Union Law and Jean Monnet Chair on European Integration at the University of Deusto (UD). PhD in Law (UD), she accomplished post-graduate studies at the Centre Européen Universitaire de Nancy, where she obtained the DESS and DEA en Droit Communautaire. She was Director of the European Studies Institute at UD from 1996 to 2009, and Academic Coordinator 
of the Jean Monnet Centre of Excellence on European Union Law and International Relations from 2013 to 2016. She also served as Vice-Dean for Research and International Relations at Deusto Faculty of Law from 2013 to 2015. She has been a visiting scholar at the Universities of Oregon (2009), Georgetown (2012), Fordham (2013), Boston College (2014) and Florida International University (2016). She is currently Editor-in- Chief of Cuadernos Europeos de Deusto Journal and Main Researcher of the Integración Europea y Derecho Patrimonial en un contexto global research team. She is author of several books on European legal issues, such as Hacia un Derecho Común en la Unión Europea (Dykinson, 2003) and El Mercado Interior Europeo (Universidad de Deusto, 2008). She has also published numerous papers in scientific journals. Her current research interests are chiefly focused on the European Union (EU)'s climate change and energy policy, democracy and citizenship in the EU, and the role of the $\mathrm{EU}$ as an international actor. 


\section{Derechos de autor}

Los derechos de autor (para la distribución, comunicación pública, reproducción e inclusión en bases de datos de indexación y repositorios institucionales) de esta publicación (Cuadernos Europeos de Deusto, CED) pertenecen a la editorial Universidad de Deusto. El acceso al contenido digital de cualquier número de Cuadernos Europeos de Deusto es gratuito inmediatamente después de su publicación. Los trabajos podrán leerse, descargarse, copiar y difundir en cualquier medio sin fines comerciales y según lo previsto por la ley; sin la previa autorización de la Editorial (Universidad de Deusto) o el autor. Así mismo, los trabajos editados en CED pueden ser publicados con posterioridad en otros medios o revistas, siempre que el autor indique con claridad y en la primera nota a pie de página que el trabajo se publicó por primera vez en $C E D$, con indicación del número, año, páginas y DOI (si procede). Cualquier otro uso de su contenido en cualquier medio o formato, ahora conocido o desarrollado en el futuro, requiere el permiso previo por escrito del titular de los derechos de autor.

\section{Copyright}

Copyright (for distribution, public communication, reproduction and inclusion in indexation databases and institutional repositories) of this publication (Cuadernos Europeos de Deusto, CED) belongs to the publisher University of Deusto. Access to the digital content of any Issue of Cuadernos Europeos de Deusto is free upon its publication. The content can be read, downloaded, copied, and distributed freely in any medium only for non-commercial purposes and in accordance with any applicable copyright legislation, without prior permission from the copyright holder (University of Deusto) or the author. Thus, the content of $C E D$ can be subsequently published in other media or journals, as long as the author clearly indicates in the first footnote that the work was published in $C E D$ for the first time, indicating the Issue number, year, pages, and DOI (if applicable). Any other use of its content in any medium or format, now known or developed in the future, requires prior written permission of the copyright holder. 